\title{
Comparisons of Inter-tablet Coating Variability under Different Tablet Shapes and Filling Levels by Using DEM Simulations
}

\author{
Qiushuang Zhang ${ }^{1, *}$, Hui Wan ${ }^{1}$, Bin Jian ${ }^{1}$, and Guoming $\mathrm{Hu}^{1}$ \\ ${ }^{1}$ School of Power and Mechanical Engineering, Wuhan University, Hubei District, Wuhan 430072, PR China
}

\begin{abstract}
The purpose of this paper is to investigate inter-tablet coating thickness variability based on different tablet shapes and filling levels. To achieve this goal, the Discrete Element Method (DEM) is applied, and efforts are made as following: For the first step, four different tablet shapes, referred to as Sphere, SRC (Standard Round Concave), Oval and Triangular, are built by importing three (except Sphere) CAD templates to create one or more spherical surfaces. Furthermore, a spray zone in the coating pan is presented to monitor the change of the "coating mass", by virtue of which we calculate the inter-tablet coating variability. And to complete this work, custom contact models and properties are created to describe coating. Then, three different filling levels are added to the simulation. Eventually, the compared results are analyzed between three diverse filling degrees among tablet particles with four discrepant shapes.
\end{abstract}

\section{Introduction}

Coating serves a wide variety of functions in pharmaceutical industry, such as avoiding the influences of external factors (moisture, air, light) and gastric acidity to guarantee the bioavailability effectiveness of drugs, masking drugs color, flavors and odors to improve the physical attribution and esthetics level of medicines and controlling the release profile of the drug to regulate its absorption degree. Consequently, controlling inter-tablet coating variability is of great significance for film coated tablets to work well. To succeed in obtaining a qualified batch of tablets, a prescribed coating with a thin film of polymer onto each tablet's surface which means low inter-tablet variability is needed.

Coating variability is divided into two types [1]: the inter-tablet coating variability which represents the coating variability from tablet to tablet, and the intra-tablet coating variability which indicates the variability in the coating mass over the surface of a single tablet. In this work, the inter-tablet variability is of particular notice to research the influential factors in the coating to minimize the inter-tablet variability.

In this paper, the Discrete Element Method (DEM) is used to simulate the coating process so as to track the inter-tablet variability. A spray zone and the spray droplets are modeled to get the coating mass in order to calculate the inter-tablet variability. Besides, an analytical work and a comparative study have been done between tablet particles in various shapes, and between several kinds of filling levels.

\section{Background}

It is a public consensus that the intra- and inter-tablet variability is two major standards that weigh coating performance. Tobiska and Kleinebudde [2] have done a research about both the intra- and inter-tablet homogeneity by using analysis of variance (ANOVA). While Mueller and Kleinebudde [3] investigated the particle motion in which the tablet velocity and surface time were included in rotating pan coaters by using imaging techniques. In recent years, DEM has been on the center stage as a popular instrument of coating process analysis. As a deluxe simulation tool with faster computation capability and stronger analytical function, it has been used frequently by many researchers in mathematical modeling of the coating effect $[4,5]$, most of whom having achieved excellent outcomes.

A multitude of scholars have taken tablet shape and pan filling into consideration to get a relatively minor numerical value of inter-tablet variability by means of experimental technique. Pandey and Turton [6] obtained a conclusion by using novel video-imaging techniques: Compared to the spherical-particles, the shaped tablets possessed faster velocity, higher dynamic angle of repose and wavier bed surface. Lower pan loads were required for minor inter-tablet coating uniformity according to Ketterhagen [7]. He also pointed out that the pan loading played a big part in the inter-tablet coating uniformity, while the tablet shape played a small one through a DEM model. Suzzi et al. [8] analyzed the influence of tablet shapes (bi-convex, oval and spherical) and filling degrees on the inter-tablet coating variability in a continuous drum.

Previous works researching inter-tablet coating variability mostly focused on tablet residence time.

*Corresponding author: $2016202080018 @$ whu.edu.cn 
Kalbag and Wassgren [9] introduced a variable quantity of coating trials to investigate the tablet residence time with a thought that there was a positive correlation between the tablet residence time and coating mass. Kumar and Wassgren [10] used a Peclet number which was defined as the ratio of axial advection rate to the axial diffusive rate to describe the inter-tablet coating variability at the premise of the residence time with a finite variance, showing a strong effect of the residence time variability on the inter-tablet coating variability.

The focus of the current work is the obtainment of the coating mass captured through an algorithm by the aid of API. So DEM simulations for four tablet shapes and three filling levels have been carried out to observe the inter-tablet coating variability in the coating process within a coating pan. A static spray zone and uniform spray droplets are applied to the DEM simulations to seek a relatively objective simulation result.

\section{Discrete element method (DEM) simulations}

\subsection{Geometry model}

The simulated tablet coating procedure in this work was operated in a Thomas Engineering Accela-Cota pan with a diameter of 24 inch $(61.0 \mathrm{~cm})$, as shown in Fig. 1. The modeled coating drum contained two baffles serving to gather the tablets to the center of the span and four anti-slip bars helping to avoid sliding which arrayed symmetrically in the cylindrical drum. Kalbag et al. [4] have given a full account of the geometry model.

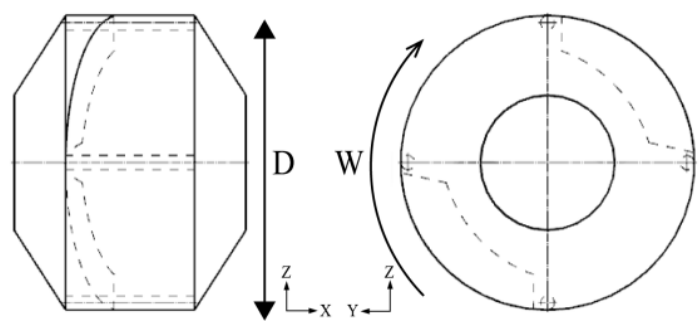

Fig.1. Diagram of the modeled coating drum. Front view (left) and side view (right)

\subsection{Tablet model}

Four different tablet shapes, including Sphere, SRC, Oval, and Triangular, were created to make out what roles they played in coating homogeneity, as shown in Fig.2. To take on a clear and all-round display, the top views of three CAD templates and a sketch of a sphere particle were presented on the upper side, and the tablet models developed by the glued spheres were displayed on the lower side for every kind of tablet shape.

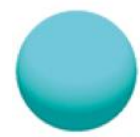

Sphere

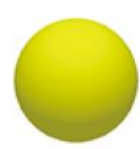

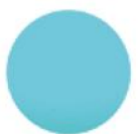

SRC

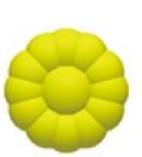

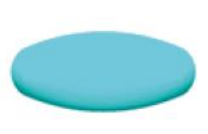

Oval

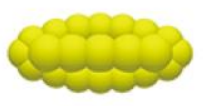

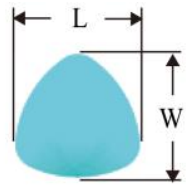

Triangular

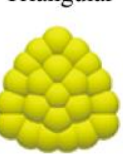

Fig. 2. Four tablet shapes (including Sphere, SRC, Oval, and Triangular) presented in the current work

The glued sphere method was applied in the current work to approach tablets' original shapes to the greatest extent by light of a clump of rigidly bonded spheres [11]. Hence, several CAD templates of tablets were established to provide references for determining the size and position of particles filling into them. The number of the constituent particles in the composite tablet shapes was chosen moderately in consideration of over-damping phenomenon (if too many) and distortion of original shapes (if too few). Otherwise, the theory that the rounder the objective particle is, the fewer spheres are demanded for tablets expression [12] was also demonstrated according to Fig. 2.

The detailed information of tablets randomly selected as representatives of differentiated shapes is illustrated in Table 1, in which several measurable parameters are given. Subsequently, the material features of the geometry as well as simulation settings indispensable for mutual contact between particle-steel and particle-particle are shown in Table 2.

\subsection{Contact model}

To reproduce the coating process, a static plane which is set to be practically vertical to the normal of the tablet bed surface is introduced in, acting as the hypothetical spray zone to produce spray droplets. For convenience, it is assumed that the spray droplets are evenly distributed within the spray zone.

To realize the spraying function, a user defined contact model is created to closely model the interactions between particles to a more accurate extent which the traditional one can not do. The approach primarily considers three contact conditions: 
Table 1. Physical properties of four kinds of tablets

\begin{tabular}{cccccc}
\hline Tablet shape & Mass $(\mathbf{g})$ & Density $(\mathbf{k g} / \mathbf{m 3})$ & Length $(\mathbf{c m})$ & Width $(\mathbf{c m})$ & Thickness $(\mathbf{c m})$ \\
\hline Triangular & 0.520 & 1300 & 1.59 & 1.57 & 0.47 \\
Oval & 0.520 & 1300 & 1.61 & 0.70 & 0.61 \\
SRC & 0.520 & 1300 & 1.08 & - & 0.70 \\
Sphere & 0.520 & 1300 & 0.85 & - & - \\
\hline
\end{tabular}

Table 2. Primary parameters set for the DEM simulation

\begin{tabular}{cc}
\hline Parameter & Value \\
\hline Physical properties-geometry(steel) & \\
\hline Pan speed & $22 \mathrm{rpm}$ \\
Poisson's ratio & 0.3 \\
Shear modulus & $7 \times 10^{10} \mathrm{~Pa}$ \\
Density & $7800 \mathrm{~kg} / \mathrm{m} 3$ \\
\hline Material properties-particle(tablet) \\
\hline Poisson's ratio \\
Shear modulus \\
\hline Contact & 0.25 \\
\hline interaction(particle-steel/particle-particle) & $1 \times 10^{6} \mathrm{~Pa}$ \\
\hline Coefficient of restitution & 0.5 \\
Coefficient of static friction & 0.5 \\
Coefficient of rolling friction & 0.01 \\
\hline
\end{tabular}

Spray droplet-spray droplet: The contact is ignored in this simulation, and the program handles with nothing and goes on next step,

Spray droplet-tablet: The mass of spray droplet is transferred to added mass of the tablet once they meet, and the spray droplet is removed at the same time,

Spray droplet-geometry: The collision is addressed in the pattern of the removal of the spray particles in this contact situation.

\section{Simulations under different filling levels and tablet shapes}

In this work, the inter-tablet coating variability is gauged according to its definition of the coefficient of variation of the coating mass between tablets [13], namely:

$$
\operatorname{CoV}_{\text {inter }}=\frac{\sigma}{\bar{m}}
$$

where $\bar{m}$, the mean coating mass over the total time, is directly obtained from the results data exported from the EDEM software depending on the contact algorithm between tablet particles and spray particles, and $\sigma$, the standard deviation, can be obtained using the following formula:

$$
\sigma=\sqrt{\frac{1}{N} \sum_{i=1}^{N}\left(m_{i}-\bar{m}\right)^{2}}
$$

Where $m_{i}$ represents the added mass that every individual tablet particle gets resulting from passing through the spray zone, and $N$ represents the total number of tablets generated in the coating drum.

As illustrated in Fig. 3, three different tablets simulations (a) Triangular (14\% filling level), (b) Oval (22\% filling level) and (c) SRC (28\% filling level) are presented. The rectangular section in green color is the hypothetical spray zone, in which spray particles are uniformly generated in a dynamic manner. Triangular, Oval and SRC tablets are presented in a way of template models created by a three-dimensional modeling software according to Fig. 3, for a particle template method is employed in this paper. The colors of tablets indicate a reflection of the amount of coating mass (red, blue and white colors stand for much, medium and little coating mass respectively) monitored by a variable quantity "mass added".
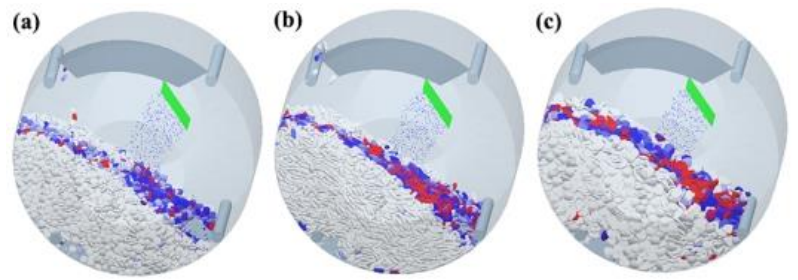

Fig. 3. Three irregular-shaping tablet simulation: (a) Triangular (14\% filling level), (b) Oval (22\% filling level) and (c) SRC (28\% filling level)

\section{Results and comparison}

Fig. 4 plots the average coating mass for each kind of tablet (Sphere, SRC, Oval and Triangular) under three different filling levels $(14 \%, 22 \%$ and $28 \%)$. It can be observed that although under different filling levels, tablets of different shapes take on similar features from Fig. 4 (a)-(c). Four kinds of tablets have an approximately linear increasing average coating mass, among which Triangular tablets possess greatest average coating mass due to the bigger surface area comparative to other different-shaping tablets, and the rest three kinds of tablets (Sphere, SRC and Oval) have close values in mean coating mass, but Oval tablets bear the least average coating mass resulting from the smaller surface area. 

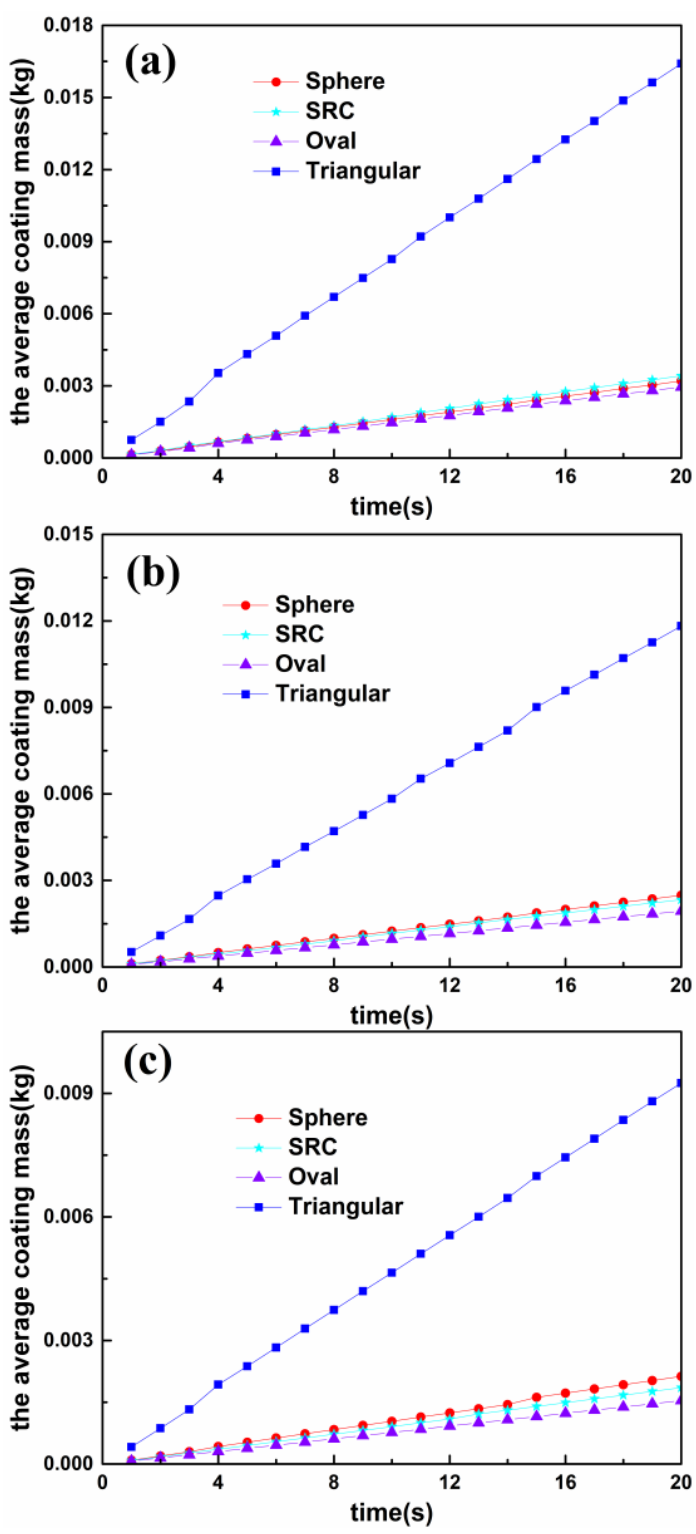

Fig. 4. Average coating mass of Sphere, and another three kinds of particles irregular in shapes, SRC, Oval, and Triangular, under three different filling levels: (a) $14 \%$ filling level; (b) 22\% filling level; (c) 28\% filling level

Fig. 5 indicates the average coating mass under $14 \%$, $22 \%$ and $28 \%$ filling levels for four kinds of irregular-shaping tablets. Fig. 5 (a)-(d) illustrates that the higher the filling level is, the less the mean coating mass attributing to the added numbers of tablets, and the bigger the digital gap is, the faster the mean coating mass decreases. It also shows that the filling ratio has the greatest influence on Triangular tablets which undulate greatly when filling level changes and the least influence on Sphere tablets which fluctuate relatively evenly on the condition of different filling ratios, and SRC and Oval tablets are impacted at the almost same level.

Fig. 6 depicts the inter-tablet coating variability $\left(C o V_{\text {inter }}\right)$ for Sphere, SRC, Oval and Triangular tablets versus time curve during simulation process under three different filling levels (14\%, 22\% and 28\%). Fig. 6 (a)-(c) indicates that $C o V_{\text {inter }}$ presents a downward trend with the simulation time getting longer no matter what kind of tablets, and finally stabilizes at a value when the time is at
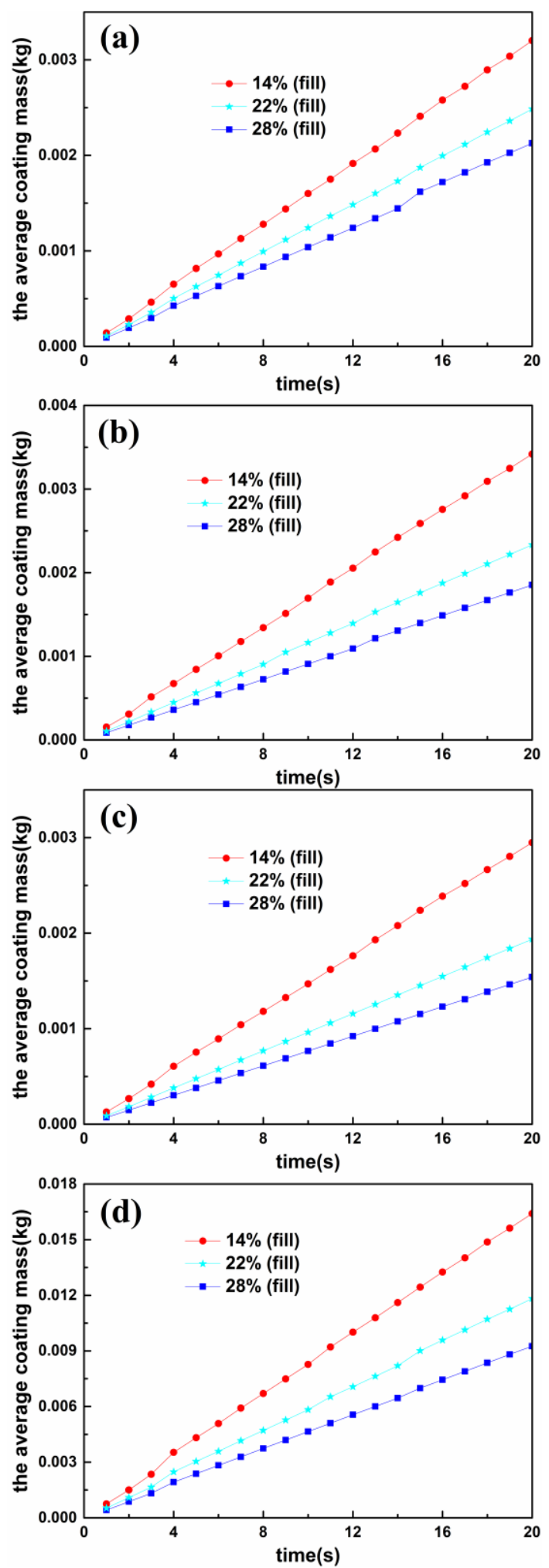

Fig. 5. Average coating mass of particles in four different shapes, (a) Sphere, (b) SRC, (c) Oval and (d) Triangular, under 14\%, $22 \%$, and $28 \%$ filling levels

$18 \mathrm{~s}$. Oval tablets get a greater $C o V_{\text {inter }}$ at a low filling level as seen from Fig. 6 (a), and a smaller $C o V_{\text {inter }}$ at high filling levels as shown in Fig. 6 (b) and (c) compared with other kinds of tablets, while Sphere tablets display contrary regularities, with the implication that Oval tablets will be a better choice when high filling levels are needed while Sphere tablets may be a reasonable option 
when low filling levels are demanded. Gaining a minimum $C o V_{\text {inter }}$ under three filling levels as shown in Fig. 6 (a)-(c), Triangular tablets exhibit a good adaptability to filling ratio, indicating that they have a good comprehensive performance.
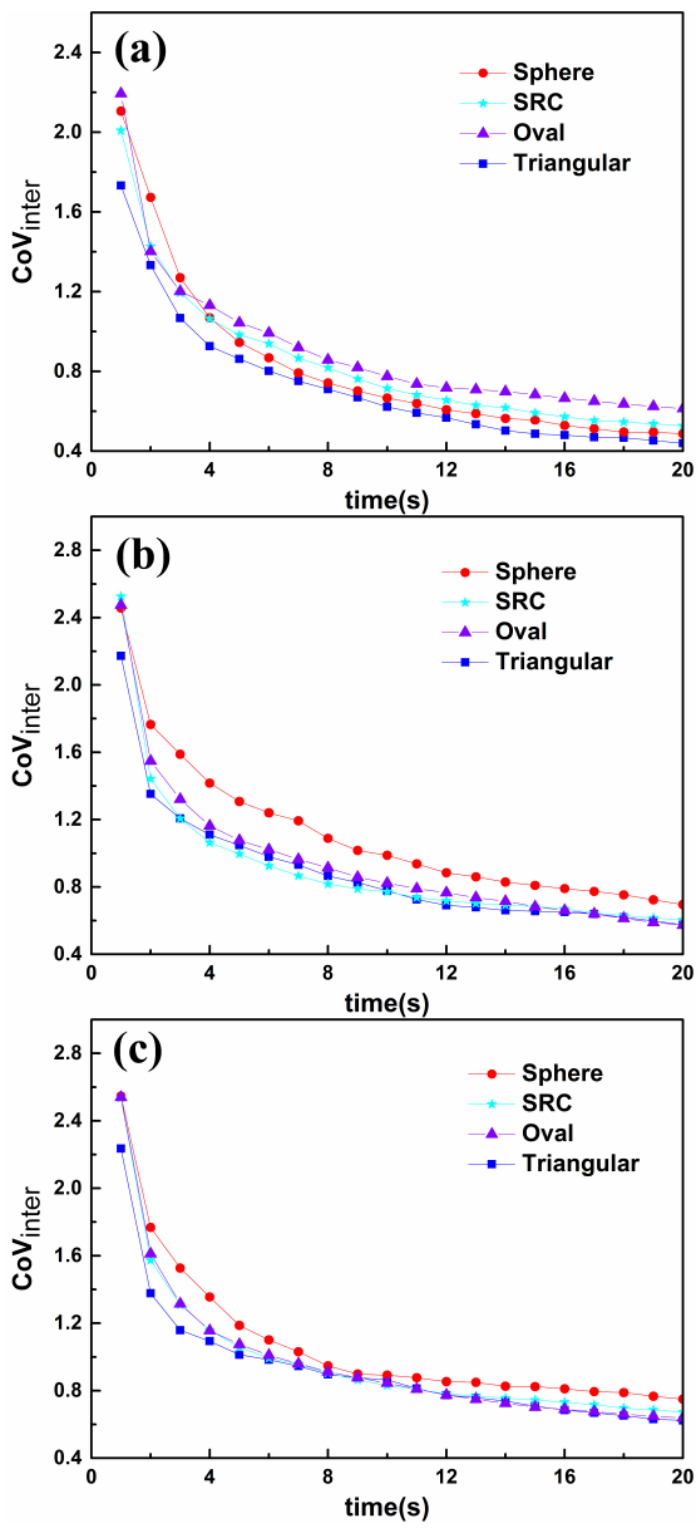

Fig. 6. Inter-tablet coating variability of Sphere, and another three kinds of particles irregular in shapes SRC, Oval, and Triangular, under three different filling levels, (a) $14 \%$ filling level, (b) 22\% filling level and (c) 28\% filling level

Fig. 7 describes the $C o V_{\text {inter }}$ versus time curve under $14 \%, 22 \%$ and $28 \%$ filling levels for each kind of tablets respectively during the whole simulation course. Contrary to the average coating mass, the higher the filling degree fraction is, the greater the $C o V_{\text {inter }}$ becomes, indicating a worse case coating uniformity. All tablets are compliant to this law, except Oval tablets, which obtain the greatest $\mathrm{CoV}_{\text {inter }}$ at $28 \%$ filling ratio, the smallest at $22 \%$, and a medium at $14 \%$, while $C o V_{\text {inter }}$ figures are very close as illustrated in Fig. 7 (c), suggesting that Oval tablets are not sensitive to filling levels but filling levels chosen for them should be prudent, because too high or too low filling levels are not fit for them either. The $C o V_{\text {inter }}$ values get a small oscillation in terms of Sphere tablets at high filling ratios, an obvious drop emerges at a low filling ratio, and a close parallel situation happens with Triangular tablets, showing that they are both propitious to low filling levels. SRC tablets demonstrate a relatively even change with the filling degree fraction getting higher.
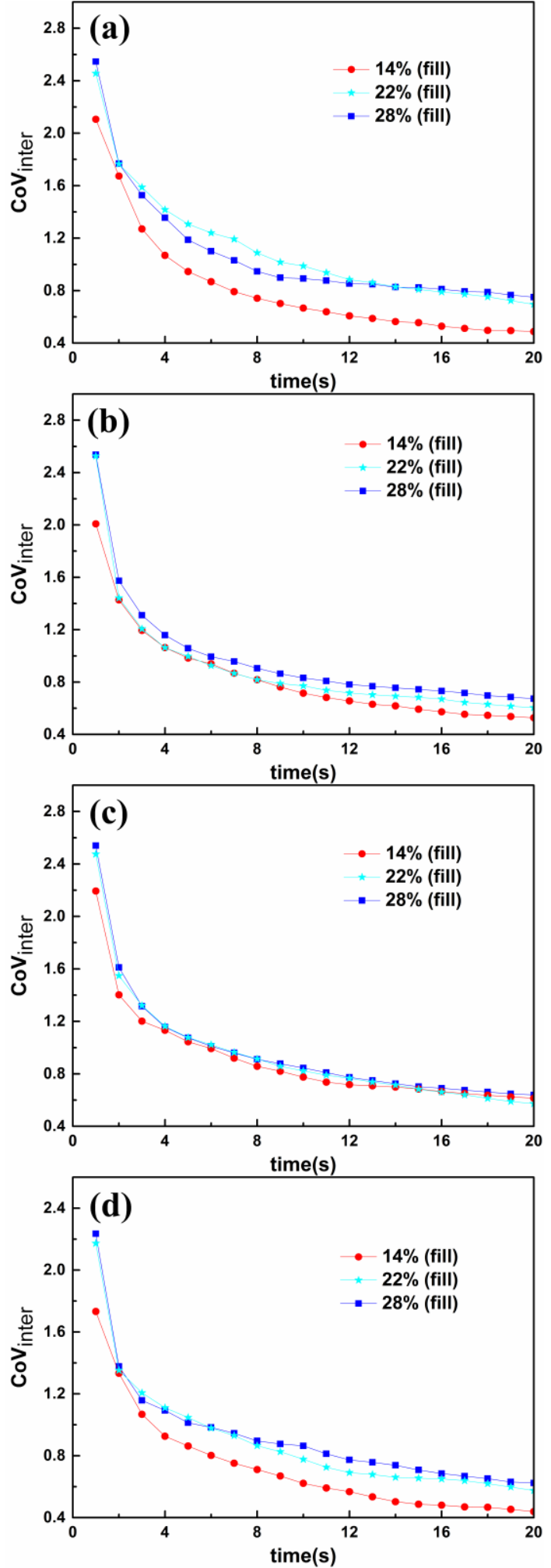

Fig. 7. Inter-tablet coating variability of particles in four different shapes, (a) Sphere, (b) SRC, (c) Oval and (d) Triangular, under $14 \%, 22 \%$, and $28 \%$ filling levels 


\section{Conclusions}

A research of inter-tablet coating thickness variability inspected from two respects, four tablet shapes (Sphere, SRC, Oval and Triangular) and three filling degrees (14\%, $22 \%$ and $28 \%$ ), in a revolving coating drum is made in this paper. Based on the special user defined contact model, the average coating mass is obtained, and finally the inter-tablet coating variability is evaluated. Through an analysis from Figs. 4-7, we come to the following conclusions:

The tablet shape acts as a decisive role in inter-tablet homogeneity. The following opinions are all concluded on the premise of comparing with the rest kinds of tablets. Triangular tablets exhibit excellent comprehensive properties, for they not only obtain the greatest average coating mass, but also the smallest $C o V_{\text {inter, which means }}$ the highest homogeneity. Sphere tablets have a bad homogeneity in high filling height condition, and a better uniformity at a low filling degree. On the contrary, in terms of Oval tablets, heterogeneity is more evident in situation of low filling levels, and a better uniformity appears in high filling degree fraction.

The filling level also has a crucial influence on the ultimate quality of the coating results. Higher filling height receives a worse coating performance in return, a conclusion in accordance with Suzzi et al. [8], in condition, it results in lower mean coating mass. Filling ratio has a greater impact on Sphere and Triangular tablets which gain a noticeably smaller homogeneity in terms of low filling height, and a tiny influence on Oval tablets, whose uniformity changes little with it.

Based on the above, we can draw a conclusion that tablet shape and filling level investigated in this paper are both indispensable considering factors to the inter-tablet coating variability. This work could serve as a reference for a better decision, choosing what kind of tablet and which filling degree. The different coater speeds and other measures investigating the coating effectiveness will be considered in further studies.

\section{Acknowledgments}

This research is supported by the National Natural Science Foundation of China (Grant No.51575404).

\section{References}

1 B. Freireich, R. Kumar, W. Ketterhagen, Chem. Eng. Sci. 131, 197-212 (2015)

2 S. Tobiska, P. Kleinebudde, Eur. J. Pharm. Biopharm. 56, 3-9 (2003)

3 R. Mueller, P. Kleinebudde, Powder Technol. 173, 51-58 (2007)

4 A. Kalbag, C. Wassgren, S.S. Penumetcha, J.D. Pérez-Ramos, Chem. Eng. Sci. 63, 2881-2894 (2008)

5 G. Toschkoff, J.G. Khinast, Eur. J. Pharm. Biopharm. 457, 407-422 (2013)

6 P. Pandey, R. Turton, AAPS PharmSciTech. 6, E237-E244 (2005)

7 W.R. Ketterhagen, Int. J. Pharm. 409, 137-149 (2011)

8 D. Suzzi, G. Toschkoff, S. Radl, D. Machold, S.D. Fraser, B.J. Glasser, J.G. Khinast, Chem. Eng. Sci. 69, 107-121 (2012)

9 R. Mueller, P. Kleinebudde, Powder Technol. 173, 51-58 (2007)

10 R. Kumar, B. Freireich, C. Wassgren, Chem. Eng. Sci. 125, 144-157 (2014)

11 M. Kodam, R. Bharadwaj, J. Curtis, B. Hancock, C. Wassgren, Chem. Eng. Sci. 64, 3466-3475 (2009)

12 M. Price, V. Murariu, G. Morrison, Fourth International Conference on Discrete Element Methods (Brisbane, Australia 2007) 